\title{
Obesity-associated miR-148a is regulated by cytokines and adipokines via a transcriptional mechanism
}

\author{
CHUNMEI SHI $^{1 *}$, LINGXIA PANG $^{1 *}$, CHENBO JI $^{1}$, JIAQIN WANG $^{2}$, NING LIN $^{3}$, JIANTAO CHEN $^{2}$, \\ LING CHEN $^{1}$, LEI YANG ${ }^{1}$, FANGYAN HUANG ${ }^{1}$, YAHUI ZHOU ${ }^{1}$, XIRONG GUO ${ }^{1}$, HUI LIANG ${ }^{4}$ and MIN ZHANG $^{1}$ \\ ${ }^{1}$ Department of Children Health Care, Nanjing Maternity and Child Health Care Hospital Affiliated to Nanjing Medical University, \\ Nanjing, Jiangsu 210029; ${ }^{2}$ Department of Pediatrics, The Third Affiliated Hospital of Xinxiang Medical College, \\ Xinxiang, Henan 453003; ${ }^{3}$ Department of Clinical Laboratory, Huai'an First People's Hospital, \\ Nanjing Medical University, Huai'an, Jiangsu 223300; ${ }^{4}$ Department of General Surgery, \\ The First Affiliated Hospital of Nanjing Medical University, Nanjing, Jiangsu 210029, P.R. China
}

Received November 3, 2015; Accepted October 7, 2016

DOI: $10.3892 / \mathrm{mmr} .2016 .5940$

\begin{abstract}
Our previous study revealed that miR-148a, a cyclic adenosine monophosphate-response element binding protein-modulated microRNA that promotes adipocyte differentiation by inhibiting Wnt1, is a biomarker of obesity in human subjects and a mouse model. The present study investigated the expression of miR-148a in human adipose tissue-derived mesenchymal stem cells (hMSCs-Ad) in response to inflammatory cytokines and adipokines to clarify its underlying mechanism. miR-148a expression was detected using reverse transcription-quantitative polymerase chain reaction analysis and its promoter activity was detected with a luciferase assay. miR-148a expression levels decreased when differentiated hMSCs-Ad were exposed to inflammatory cytokines or adipokines, which suggested that miR-148a may be important in adipocyte metabolism and inflammation. Furthermore, the promoter activity of miR-148a decreased following treatment of cells with inflammatory cytokines or adipokines. The results
\end{abstract}

Correspondence to: Dr Min Zhang, Department of Children Health Care, Nanjing Maternity and Child Health Care Hospital Affiliated to Nanjing Medical University, 123 Tianfei Road, Nanjing, Jiangsu 210029, P.R. China

E-mail: ntzhangmin@163.com

Dr Hui Liang, Department of General Surgery, The First Affiliated Hospital of Nanjing Medical University, 1 Zhongfu Road, Nanjing, Jiangsu 210029, P.R. China

E-mail: drhuiliang@126.com

*Contributed equally

Abbreviations: CREB, cyclic adenosine monophosphate-response element binding protein; MSCs, mesenchymal stem cells; hMSCs-Ad, human adipose tissue-derived mesenchymal stem cells; IL, interleukin; TNF, tumor necrosis factor

Key words: miR-148a, inflammatory cytokines, adipokines, human adipose tissue-derived mesenchymal stem cells, obesity of the present study indicated a novel role of miR-148a in adipocyte inflammation; therefore, miR-148a may be involved in obesity complications via its own underlying transcriptional mechanism.

\section{Introduction}

Obesity rates are increasing worldwide, and its incidence has risen extensively in the last three decades (1). Obesity is an established risk factor for metabolic diseases, including insulin resistance, type 2 diabetes mellitus, hypertension, nonalcoholic fatty liver disease and various cancers (1).

Obesity is associated with a chronic low-grade inflammatory state (2). In obese individuals, adipocytes synthesize and secrete large quantities of cytokines, including interleukin (IL)-6 and tumor necrosis factor $\alpha(\mathrm{TNF}-\alpha)$, adipokines, including the hormones leptin and resistin, and chemokines, which cause the migration of inflammatory cells into adipose tissue. The abnormal release of hormones, cytokines and chemokines by adipose tissue affects insulin sensitivity in an endocrine manner in the liver and skeletal muscle and in an auto-/paracrine fashion in adipose tissue. Previous studies have demonstrated that insulin resistance and obesity are closely associated with adipose tissue inflammation $(3,4)$, indicating that suppressing adipocyte inflammation may have a beneficial effect on insulin sensitivity in obese individuals $(5,6)$. In contrast, a review by Ye and McGuinness (4) suggested that elevation of proinflammatory cytokines increases energy expenditure and decreases the risk of obesity, indicating that proinflammatory cytokines may have beneficial effects.

ILs, including IL-6 and IL-1 $\beta$, affect adipocyte function, contributing to insulin resistance due to obesity. IL-6 is abundantly expressed by adipose tissue and a negative correlation has been demonstrated in humans between plasma IL-6 levels and insulin sensitivity (7). In addition, IL-6 has been revealed to be important for mesenchymal stem cell (MSC) inflammatory function $(8,9)$. MSCs have a immunoregulatory capacity, which may be induced by certain combinations of inflammatory cytokines, including interferon- $\gamma$ (IFN $\gamma)$ and $\mathrm{TNF} \alpha$, or IFN $\gamma$ and IL-1 (10). 
miR-148a was identified as a DNA methylation-associated silencing tumor suppressor involved in human cancer metastasis (11). Adipocytes are continuously stimulated by proinflammatory cytokines, including TNF- $\alpha$ and IL-6, which contribute to the inflammatory response and result in adipocyte dysfunction. Studies by our laboratory and others have demonstrated that miR-148a is an important regulator that contributes to adipogenesis via targeting Wnt1 (12) and DNA methyltransferase 1 (13). The majority of previous studies have focused on the regulation of miR-148a expression by inflammation in non-adipocytes (14). Therefore, little is known regarding the underlying mechanisms that regulate miR-148a expression during the obesity-induced inflammatory response.

The present study examined the expression of miR-148a in differentiated human adipose tissue-derived MSCs (hMSCs-Ad) and their response to proinflammatory cytokines. miR-148a transcription was decreased in inflammatory and insulin resistance microenvironments. The core promoter region of miR-148a contains cyclic adenosine monophosphate-response element binding protein (CREB) binding sites and various nuclear receptor response elements, including the CCAAT enhancer binding protein and E2F transcription factor. In addition, the CREB binding site is in the core promoter region of miR-148a (12). A luciferase assay revealed that CREB binding to the miR-148a promoter region was decreased following TNF- $\alpha$ or IL- 6 treatment, indicating that differentiated hMSCs-Ad responded to inflammatory cytokines by decreasing miR-148a expression, which may have resulted from an effect on the promoter activity. The results of the present study indicated that the inhibitionn of miR-148a during the proinflammatory response is regulated by a transcriptional event.

\section{Materials and methods}

Cell culture and adipocyte differentiation. hMSCs-Ad cells were obtained from ScienCell Research Laboratories (Carlsbad, CA, USA) and maintained in MSC medium (MSCM; ScienCell Research Laboratories) supplemented with 5\% fetal bovine serum (FBS, ScienCell Research Laboratories), 1\% MSC growth supplement (ScienCell Research Laboratories) and $1 \%$ penicillin/streptomycin solution, at $37^{\circ} \mathrm{C}$ and $5 \%$ $\mathrm{CO}_{2}$. To induce differentiation, the hMSCs-Ad were cultured in serum-free MSCM supplemented with $50 \mathrm{nM}$ insulin, $100 \mathrm{nM}$ dexamethasone, $0.5 \mathrm{mM}$ 3-isobutyl-1-methylxanthine and $100 \mu \mathrm{M}$ rosiglitazone (all from Sigma-Aldrich; Merck Millipore, Darmstadt, Germany) (day 0) and the medium was replaced every 2 days for 4 days. Subsequently, cells were cultured in serum-free MSCM supplemented with $50 \mathrm{nM}$ insulin; medium was replaced every 2 days until lipid accumulation occurred (day 10).

Treatment with cytokines/adipokines. Experiments were performed using differentiated adipocytes, 15 days following the induction of differentiation. At this time point $>80 \%$ of cells exhibited the morphological and biochemical properties of adipocytes. Following an overnight incubation in serum-free MSCM, cells were treated with 5,10 or $20 \mathrm{ng} / \mathrm{ml} \mathrm{TNF-} \alpha$ (Merck Millipore) (15), 10, 30 or 90 ng/ml IL-6 (Merck
Millipore) (16), $30 \mathrm{ng} / \mathrm{ml}$ leptin or $60 \mathrm{ng} / \mathrm{ml}$ resistin (Merck Millipore) for 4,8 or $24 \mathrm{~h}$. Cells were harvested at these time points for subsequent experiments.

$R N A$ isolation and reverse transcription-quantitative polymerase chain reaction ( $R T-q P C R)$. Total RNA was prepared from hMSCs-Ad at various time points following adipocyte differentiation induction using TRIzol ${ }^{\circledR}$ reagent (Invitrogen; Thermo Fisher Scientific, Inc., Waltham MA, USA) according to the manufacturer's protocol, followed by DNase treatment (Takara Bio, Inc., Otsu, Japan). The quality and quantity of RNA was assessed using a NanoDrop 2.0 (Thermo Fisher Scientific, Inc.). cDNA was synthesized from 200 ng RNA using the TaqMan MicroRNA Reverse Transcription kit (Applied Biosystems; Thermo Fisher Scientific, Inc.). TaqMan Mix (Applied Biosystems; Thermo Fisher Scientific, Inc.) and miRNA probe (Applied Biosystems; Thermo Fisher Scientific, Inc.) were used to amplify the cDNA by qPCR. qPCR was performed using an Applied Biosystems 7500 Sequence Detection system (Applied Biosystems; Thermo Fisher Scientific, Inc.) according to the manufacturer's protocol. Cycling conditions were as follows: An initial denaturation step at $95^{\circ} \mathrm{C}$ for $10 \mathrm{~min}$ was followed by 40 cycles of denaturation at $95^{\circ} \mathrm{C}$ for $15 \mathrm{sec}$ and annealing at $60^{\circ} \mathrm{C}$ for $1 \mathrm{~min}$. miR-148a expression was normalized to snoU6. The primer identification numbers are 000470 (miR-148a) and 001973 (snoU6; Applied Biosystems; Thermo Fisher Scientific, Inc.). Each sample was measured in triplicate, and the mRNA expression levels were calculated using the $2^{-\Delta \Delta \mathrm{cq}}$ method (17).

Promoter reporter assays. The miR-148a promoter and control pGL3-basic promoter (Promega Corporation, Madison, WI, USA) were established in our previous study (12). Human embryonic kidney 293T (HEK293T; American Type Culture Collection, Manassas, VA, USA) cells were cultured in Dulbecco's modified Eagle's medium supplemented with $10 \%$ FBS and 4 mM L-glutamine. HEK293T cells were cultured to $60-70 \%$ confluence in 6-well plates exposed to cytokines/adipokines. Promoter activity was assessed using the Dual-Glo ${ }^{\circledR}$ Luciferase assay system (Promega Corporation). Cells were transfected with $250 \mathrm{ng} /$ well promoter-Firefly luciferase reporter construct and $25 \mathrm{ng} /$ well Renilla luciferase vector (pRL-TK) using $0.6 \mu 1$ Lipofectamine ${ }^{\circledR} 2000$ (Thermo Fisher Scientific, Inc.) in $20 \mu$ l Opti-minimal essential medium ${ }^{\circledR}$ I Reduced Serum (Thermo Fisher Scientific, Inc.). A total of $24 \mathrm{~h}$ later, cells were lysed in $50 \mu \mathrm{l} 1 \mathrm{X}$ Passive Lysis buffer (Promega Corporation) and stored at $-20^{\circ} \mathrm{C}$ until analysis. Assays were performed in quadruplicate and repeated three times.

Statistical analysis. SPSS software version 17.0 (SPSS, Inc., Chicago, IL, USA) was used for statistical analysis. Representatives of replicate experiments are presented in the figures and the data are expressed as the mean \pm standard error. Differences between groups were analyzed using Student's two-tailed $t$-test when two groups were compared, or one-way analysis of variance followed by the least significant difference post hoc test when multiple groups were compared. $\mathrm{P}<0.05$ was considered to indicate a statistically significant difference. 


\section{Results}

miR -148 a is regulated by the adipokines leptin and resistin in differentiated hMSCs-Ad. Fig. 1 presents a schematic representation of the chromosomal location of miR-148a. Our previous study revealed that miR-148a was highly expressed in mature adipocytes using a microRNA chip (12). To investigate the effects of adipokines on the expression of miR-148a, the present study simulated the adipokine microenvironment by exposing hMSCs-Ad to leptin or resistin. Differentiated hMSCs-Ad were treated with $30 \mathrm{ng} / \mathrm{ml}$ leptin or $60 \mathrm{ng} / \mathrm{ml}$ resistin; miR-148a expression was examined at 4,8 and $24 \mathrm{~h}$ and normalized to snoU6 expression. miR-148a expression levels were significantly decreased at all time points following treatment with leptin $(\mathrm{P}<0.001$ at all time points; Fig. $2 \mathrm{~A})$ or resistin $(\mathrm{P}=0.002,4 \mathrm{~h}$ vs. $0 \mathrm{~h} ; \mathrm{P}=0.003,8 \mathrm{~h}$ vs. $0 \mathrm{~h} ; \mathrm{P}=0.001$, 24 h vs. 0 h; Fig. 2B).

miR-148a is regulated by IL-6 in hMSCs-Ad. The effect of the inflammatory cytokine IL-6 on miR-148a expression levels in hMSCs-Ad was assessed. Differentiated hMSCs-Ad were treated with IL-6, as in a previous study (16), and the miR-148a expression level was examined at 4, 8 and $24 \mathrm{~h}$ and normalized to snoU6 expression. miR-148a expression levels were significantly decreased following treatment with low dose (10 ng/ml; $\mathrm{P}=0.004$, 4 h vs. 0 h; $\mathrm{P}<0.001,8$ h vs. 0 h; $\mathrm{P}<0.001$, $24 \mathrm{~h}$ vs. 0 h; Fig. $3 \mathrm{~A})$, moderate dose $(30 \mathrm{ng} / \mathrm{ml} ; \mathrm{P}=0.008,4 \mathrm{~h}$ vs. 0 h; $\mathrm{P}<0.001,8$ h vs. 0 h; $\mathrm{P}<0.001,24$ h vs. 0 h; Fig. $3 \mathrm{~B})$ and high dose (90 ng/ml; $\mathrm{P}<0.001$ at all time points; Fig. $3 \mathrm{C}$ ) IL-6, at all time points. miR-148a expression levels were significantly reduced at $24 \mathrm{~h}$ of $10 \mathrm{ng} / \mathrm{ml}$ IL- 6 stimulation, by $>80 \%$ compared with the control (Fig. 3A). However, IL-6 did not decrease miR-148a expression levels in a dose-dependent manner.

Effect of TNF- $\alpha$ on miR-148a expression levels in hMSCs-Ad. In addition, the effect of treatment with low dose $(5 \mathrm{ng} / \mathrm{ml}$; $\mathrm{P}<0.001$ at all time points; Fig. 3D), moderate dose $(10 \mathrm{ng} / \mathrm{ml}$; $\mathrm{P}=0.017,4$ h vs. 0 h; $\mathrm{P}=0.005,8$ h vs. 0 h; $\mathrm{P}=0.003,24$ h vs. 0 h; Fig. 3E) and high dose $(20 \mathrm{ng} / \mathrm{ml} ; \mathrm{P}<0.001$ at all time points; Fig. 3F) TNF- $\alpha$ was examined. miR-148a expression levels in differentiated hMSCs-Ad treated with $10 \mathrm{ng} / \mathrm{ml} \mathrm{TNF-} \alpha$, as in a previous study (15), were significantly downregulated compared with the control at $8(\mathrm{P}=0.005$; Fig. $3 \mathrm{E})$ and $24 \mathrm{~h}$ $(\mathrm{P}=0.003$; Fig. 3E). However, miR-148a expression levels were not decreased in a dose-dependent manner. Exposure of hMSCs-Ad to TNF- $\alpha$ and IL- 6 resulted in decreased miR-148a expression levels, suggesting that the obesity-associated inflammatory microenvironment inhibited miR-148a expression.

Decreased promoter activity in the primary promoter of miR-148a as a result of inflammatory cytokine or adipokine treatment. Our previous study determined that the primary promoter of miR-148a is located at the promoter region-2947 to-2687 nt of pre-miR-148a (12). To clarify the underlying mechanism by which adipokines and cytokines regulate miR-148a expression, HEK293T cells were used to analyze the effect of IL-6 $(\mathrm{P}<0.001$; Fig. 4A), TNF- $\alpha(\mathrm{P}=0.002$; Fig. 4B), leptin $(\mathrm{P}<0.001$; Fig. $4 \mathrm{C})$ and resistin $(\mathrm{P}<0.001$; Fig. 4D) on the primary promoter activity of the gene encoding
Chromosome [7]

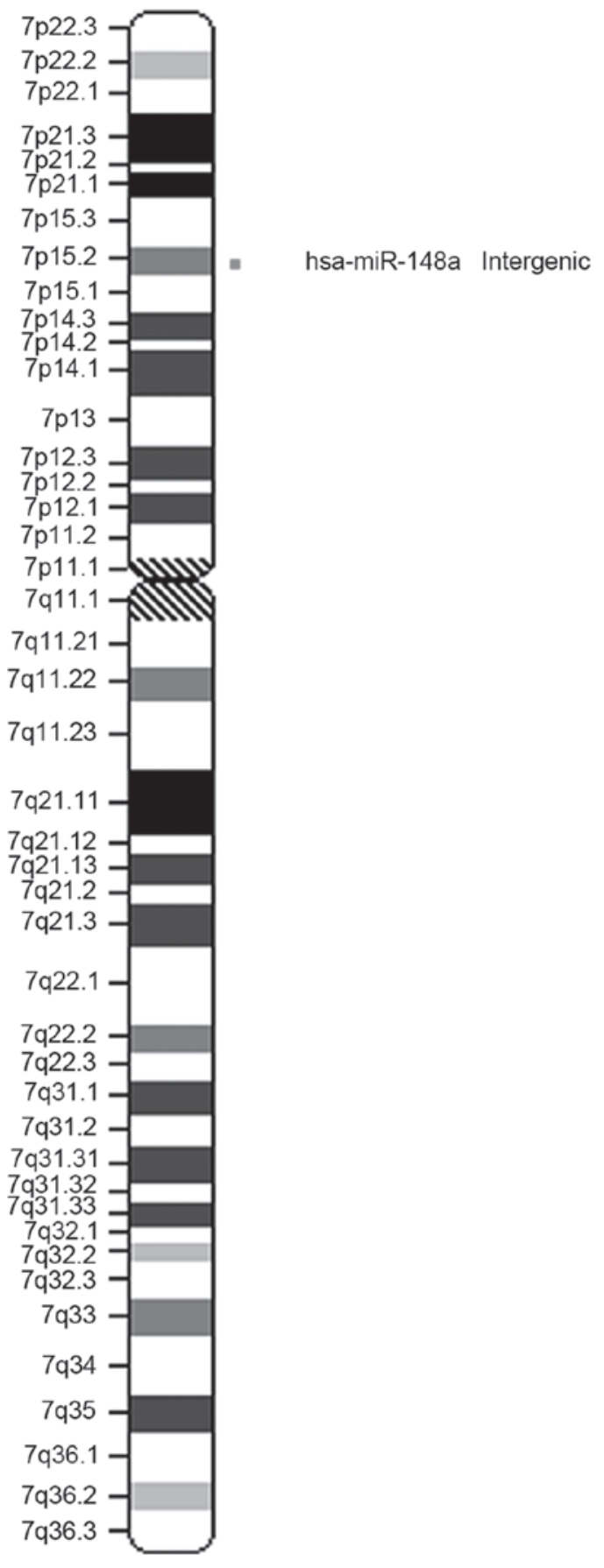

Figure 1. Schematic representation of the chromosomal location of miR-148a.

miR-148a. miR-148a promoter activity was significantly reduced by $\sim 60 \%$ (TNF- $\alpha$ and resistin) or $\sim 70 \%$ (IL-6 and leptin) compared with untreated cells $(\mathrm{P}<0.001)$, which was normalized to pGL3-basic. A luciferase assay revealed that the inflammatory cytokines decreased miR-148a promoter activity, and this effect was consistent with inflammatory cytokine levels that inhibited miR-148a at the transcriptional level (Fig. 3). Therefore, miR-148a expression levels were decreased in differentiated hMSCs-Ad in response to treatment with inflammatory cytokines, which may be the result of a decrease in the miR-148a promoter activity. 

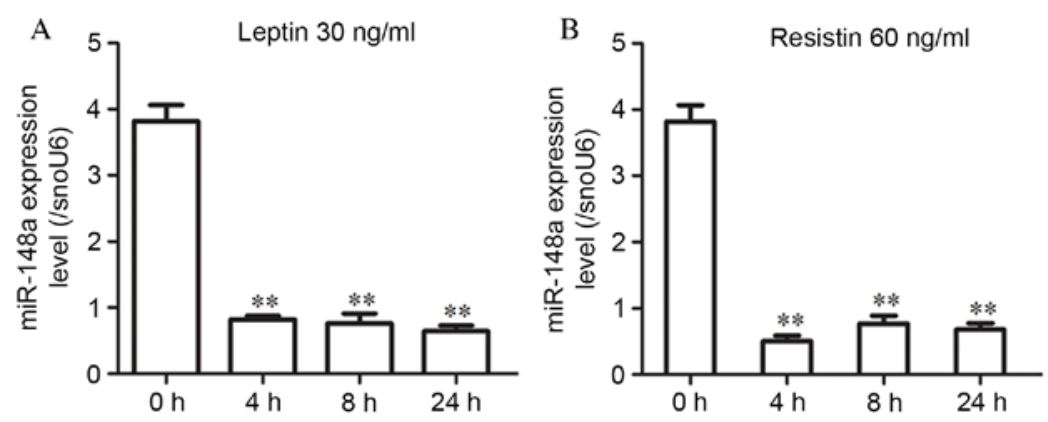

Figure 2. miR-148a expression levels in differentiated hMSCs-Ad are regulated by leptin and resistin. Cells were treated with (A) $30 \mathrm{ng} / \mathrm{ml}$ leptin or (B) $60 \mathrm{ng} / \mathrm{ml}$ resistin. Following 4, 8 or $24 \mathrm{~h}$ of incubation, miR-148a expression levels were analyzed by reverse transcription-quantitative polymerase chain reaction and normalized to snoU6 levels. miR-148a expression levels were significantly decreased by leptin or resistin treatment at all time points. Data are expressed as the mean \pm standard error $(n=3)$ and are representative of three independent experiments. ${ }^{* *} \mathrm{P}<0.01$ vs. control (untreated cells, $0 \mathrm{~h}$ ). miR, microRNA; hMSCs-Ad, human adipose tissue-derived mesenchymal stem cells.
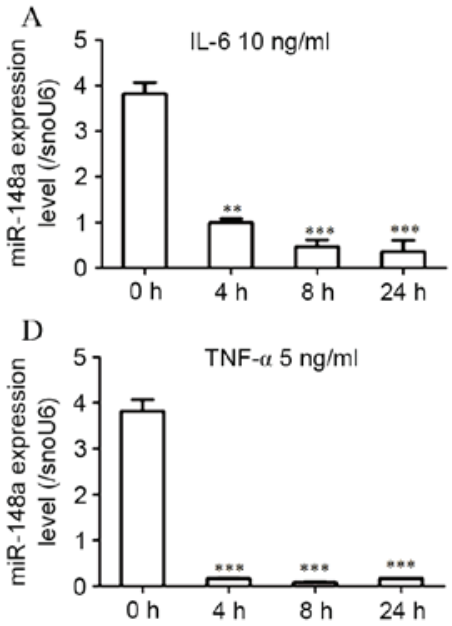
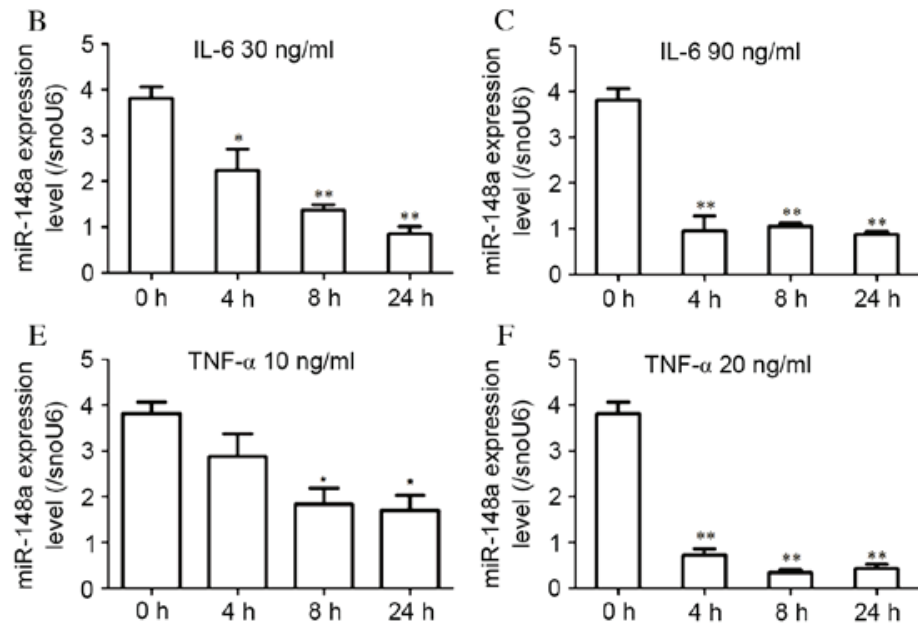

Figure 3. miR-148a expression levels in hMSCs-Ad are regulated by IL-6 and TNF- $\alpha$. Cells were treated with (A) 10, (B) 30 or (C) 90 ng/ml IL-6, or (D) 5, (E) 10 or (F) $20 \mathrm{ng} / \mathrm{ml}$ TNF- $\alpha$. Following 4,8 or $24 \mathrm{~h}$ of incubation, miR-148a expression levels were analyzed by reverse transcription-quantitative polymerase chain reaction and normalized to snoU6 levels. miR-148a expression levels were significantly decreased by IL- 6 or TNF- $\alpha$; however, this effect was not dose-dependent. Data are expressed as the mean \pm standard error $(\mathrm{n}=3)$ and are representative of three independent experiments. ${ }^{*} \mathrm{P}<0.05,{ }^{* * *} \mathrm{P}<0.01$ and ${ }^{* * * *} \mathrm{P}<0.001$ vs. control (untreated cells, 0 h). miR, microRNA; hMSCs-Ad, human adipose tissue-derived mesenchymal stem cells; IL, interleukin; TNF, tumor necrosis factor.
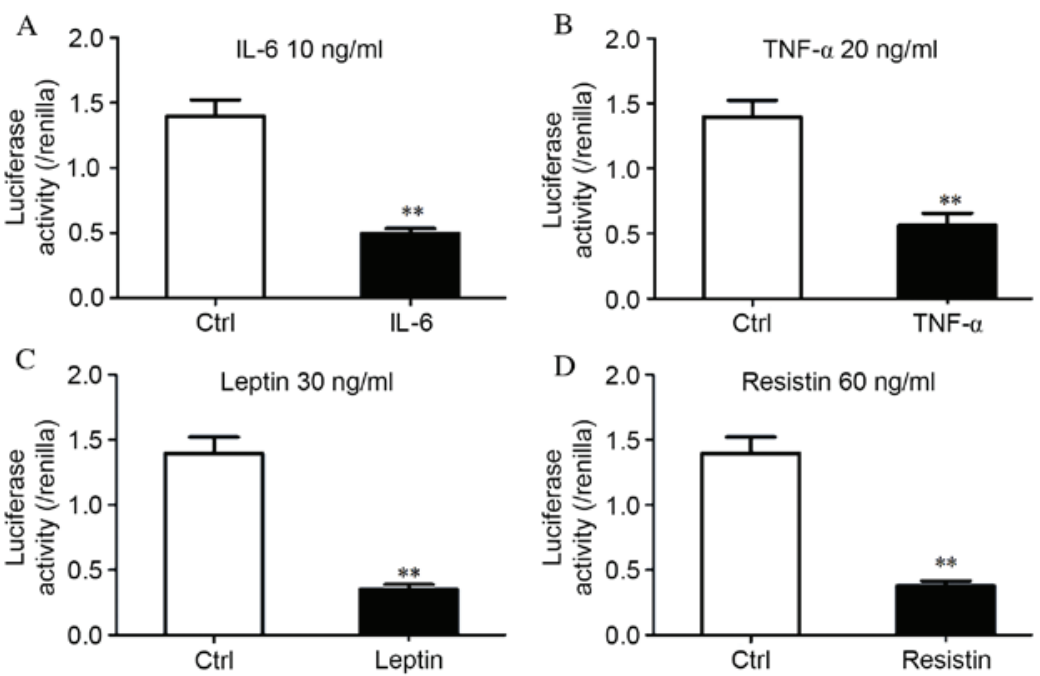

Figure 4. miR-148a promoter activity in HEK293T cells is decreased by inflammatory cytokines and adipokines. Cells were transfected with promoter-luciferase constructs of miR-148a (the core promoter) and empty construct as the control, and treated with (A) $10 \mathrm{ng} / \mathrm{ml} \mathrm{IL-6,} \mathrm{(B)} 20 \mathrm{ng} / \mathrm{ml} \mathrm{TNF}-\alpha$, (C) $30 \mathrm{ng} / \mathrm{ml}$ leptin or (D) $60 \mathrm{ng} / \mathrm{ml}$ resistin. The promoter activity was estimated by measuring the luciferase activity. miR-148a promoter activity was significantly reduced by treatment with all 4 molecules, compared with untreated cells. Data are expressed as the mean \pm standard error $(n=4)$ and are representative of three independent experiments. ${ }^{* *} \mathrm{P}<0.01$ vs. control. miR, microRNA; Ctrl, control; IL, interleukin; TNF, tumor necrosis factor. 


\section{Discussion}

Although obesity has been associated with inflammatory cytokine accumulation and altered insulin sensitivity of adipocytes, these concepts remain controversial. miR-148a was recently characterized as a novel obesity-associated microRNA with a role in adipogenesis and energy metabolism $(12,13)$. Studies by our laboratory and others revealed miR-148a as an important regulator involved in adipogenesis via targeting Wnt1 $(12,18)$, and demonstrated that miR-148a was highly expressed in obese individuals (12). However, the metabolic regulation of miR-148a in adipocytes has not been comprehensively investigated. The present study investigated the expression levels of miR-148a in hMSCs-Ad in response to inflammatory cytokines and adipokines.

Certain studies have demonstrated that the adipokines leptin and resistin are involved in obesity-associated insulin resistance as well as in adipocyte differentiation (19,20). Leptin, an adipocyte-derived hormone and cytokine, is upregulated in patients with obesity-associated type 2 diabetes mellitus, although leptin resistance has been reported (21). However, the association between miR-148a and adipokines remains to be fully elucidated. The present study investigated the regulation of miR-148a expression in adipocytes modulated by adipokines. Leptin and resistin markedly downregulated miR-148a expression levels. Our previous study revealed that miR-148a was overexpressed in obese individuals (12). Therefore, leptin and resistin may suppress miR-148a expression through negative feedback; however, this association requires further investigation.

IL- 6 has been described as a resolution factor, which affects the immune system by balancing inflammatory and anti-inflammatory responses (22). In addition, IL-6 is an important cytokine with extensive biological activities in processes including immune regulation, inflammation, hematopoiesis and oncogenesis. TNF- $\alpha$ has numerous adverse effects on adipocyte functions, including increased basal lipolysis and reduced insulin sensitivity, as reviewed in (23). IL-6 and TNF- $\alpha$ additionally modulate miR-148a expression levels. The present study revealed that miR-148a expression levels were decreased in differentiated hMSCs-Ad treated with IL-6. However, this effect was not dose-dependent. This may be due to the fact that miR-148a is highly expressed in differentiated hMSC-Ad. Alternatively, there may be other compensatory mechanisms involved. These findings were consistent with a previous study that demonstrated that miR-148a expression levels were decreased in IL-6-overexpressing malignant cholangiocytes in vitro, and in tumor cell xenografts (14). In the present study, TNF- $\alpha$ significantly downregulated miR-148a expression compared with the control, suggesting that obesity-associated inflammation inhibits miR-148a expression.

Our previous study determined that the primary miR-148a promoter is located at the promoter region -2947 to $-2687 \mathrm{nt}$ of pre-miR-148a (12). Therefore, the present study investigated whether the primary promoter was involved in the downregulation of miR-148a by inflammatory cytokines and adipokines. miR-148a promoter activity was significantly reduced following treatment with IL- 6 , TNF- $\alpha$, leptin and resistin compared with the control. These results suggested that miR-148a expression levels were decreased in differ- entiated hMSCs-Ad in response to inflammatory cytokines and adipokines, potentially due to an effect on its promoter activity.

A previous study revealed that adipocyte inflammation is essential for healthy adipose tissue expansion and remodeling (24); this does not contradict results from a model in which chronic inflammation is an important contributor toward metabolic syndrome (25). In conclusion, the results of the present study revealed the miR-148a expression levels and promoter activity are regulated by inflammatory cytokines and adipokines. These findings suggested a novel role for miR-148a in adipocyte inflammation, indicating that miR-148a may be involved in obesity complications via its own underlying transcriptional mechanism.

\section{Acknowledgements}

The present study was supported by grants from the National Key Basic Research Program of China (grant no. 2013CB530604), the Key Project of the National Natural Science Foundation of China (grant no. 81330067), the National Natural Science Foundation of China (grant nos. 81270928, 81370964 and 81500674), the National Natural Science Foundation of Jiangsu Province (grant no. BK20150082), the Program for Innovative Research Teams of Jiangsu Province (grant no. LJ201108), the 333 High Level Talents Training Project of Jiangsu Province, the Nanjing Technological Development Program (grant no. 201104013) and the Science and Technology Development Fund of Nanjing Medical University (grant no. 2014NJMUZD041).

\section{References}

1. Ogden CL, Carroll MD, Lawman HG, Fryar CD, Kruszon-Moran D, Kit BK and Flegal KM: Trends in obesity prevalence among children and adolescents in the United States, 1988-1994 Through 2013-2014. JAMA 21: 2292-2299, 2016.

2. Glass CK and Olefsky JM: Inflammation and lipid signaling in the etiology of insulin resistance. Cell Metab 15: 635-645, 2012.

3. Patsouris D, Li PP, Thapar D, Chapman J, Olefsky JM and Neels JG: Ablation of CD11c-positive cells normalizes insulin sensitivity in obese insulin resistant animals. Cell Metab 8: 301-309, 2008.

4. Ye J and McGuinness OP: Inflammation during obesity is not all bad: Evidence from animal and human studies. Am J Physiol Endocrinol Metab 304: E466-E477, 2013.

5. Osborn $\mathrm{O}$ and Olefsky JM: The cellular and signaling networks linking the immune system and metabolism in disease. Nat Med 18: 363-374, 2012.

6. Romeo GR, Lee J and Shoelson SE: Metabolic syndrome, insulin resistance, and roles of inflammation-mechanisms and therapeutic targets. Arterioscler Thromb Vasc Biol 32: 1771-1776, 2012.

7. Kern PA, Ranganathan S, Li C, Wood L and Ranganathan G: Adipose tissue tumor necrosis factor and interleukin-6 expression in human obesity and insulin resistance. Am J Physiol Endocrinol Metab 280: E745-E751, 2001.

8. Xu G, Zhang Y, Zhang L, Ren G and Shi Y: The role of IL-6 in inhibition of lymphocyte apoptosis by mesenchymal stem cells. Biochem Biophys Res Commun 361: 745-750, 2007.

9. Djouad F, Charbonnier LM, Bouffi C, Louis-Plence P, Bony C, Apparailly F, Cantos C, Jorgensen C and Noël D: Mesenchymal stem cells inhibit the differentiation of dendritic cells through an interleukin-6-dependent mechanism. Stem Cells 25: 2025-2032, 2007.

10. Ren G, Zhang L, Zhao X, Xu G, Zhang Y, Roberts AI, Zhao RC and Shi Y: Mesenchymal stem cell-mediated immunosuppression occurs via concerted action of chemokines and nitric oxide. Cell Stem Cell 2: 141-150, 2008. 
11. Lujambio A,CalinGA,VillanuevaA,RoperoS,Sánchez-CéspedesM, Blanco D, Montuenga LM, Rossi S, Nicoloso MS, Faller WJ, et al: A microRNA DNA methylation signature for human cancer metastasis. Proc Natl Acad Sci USA 105: 13556-13561, 2008.

12. Shi C, Zhang M, Tong M, Yang L, Pang L, Chen L, Xu G, Chi X Hong Q, Ni Y, et al: miR-148a is associated with obesity and modulates adipocyte differentiation of mesenchymal stem cells through Wnt signaling. Sci Rep 5: 9930, 2015.

13. Londoño Gentile T, Lu C, Lodato PM, Tse S, Olejniczak SH Witze ES, Thompson CB and Wellen KE: DNMT1 is regulated by ATP-citrate lyase and maintains methylation patterns during adipocyte differentiation. Mol Cell Biol 33: 3864-3878, 2013.

14. Braconi C, Huang N and Patel T: MicroRNA-dependent regulation of DNA methyltransferase-1 and tumor suppressor gene expression by interleukin-6 in human malignant cholangiocytes. Hepatology 51: 881-890, 2010.

15. Wellen KE, Fucho R, Gregor MF, Furuhashi M, Morgan C, Lindstad T, Vaillancourt E, Gorgun CZ, Saatcioglu F and Hotamisligil GS: Coordinated regulation of nutrient and inflammatory responses by STAMP2 is essential for metabolic homeostasis. Cell 129: 537-548, 2007.

16. Kralisch S, Klein J, Lossner U, Bluher M, Paschke R, Stumvoll M and Fasshauer M: Interleukin-6 is a negative regulator of visfatin gene expression in 3T3-L1 adipocytes. Am J Physiol Endocrinol Metab 289: E586-E590, 2005.

17. Livak KJ and Schmittgen TD: Analysis of relative gene expression data using real-time quantitative PCR and the 2(-Delta Delta C(T)) method. Methods 25: 402-408, 2001.
18. Qin L, Chen Y, Niu Y, Chen W, Wang Q, Xiao S, Li A, Xie Y, Li J, Zhao X, et al: A deep investigation into the adipogenesis mechanism: Profile of microRNAs regulating adipogenesis by modulating the canonical Wnt/beta-catenin signaling pathway. BMC Genomics 11: 320, 2010.

19. Steppan CM, Bailey ST, Bhat S, Brown EJ, Banerjee RR, Wright CM, Patel HR, Ahima RS and Lazar MA: The hormone resistin links obesity to diabetes. Nature 409: 307-312, 2001.

20. Kim KH, Lee K, Moon YS and Sul HS: A cysteine-rich adipose tissue-specific secretory factor inhibits adipocyte differentiation. J Biol Chem 276: 11252-11256, 2001.

21. Maffei M, Halaas J, Ravussin E, Pratley RE, Lee GH, Zhang Y, Fei H, Kim S, Lallone R and Ranganathan S: Leptin levels in human and rodent: Measurement of plasma leptin and ob RNA in obese and weight-reduced subjects. Nat Med 1: 1155-1161, 1995.

22. Jones SA: Directing transition from innate to acquired immunity: Defining a role for IL-6. J Immunol 175: 3463-3468, 2005.

23. Sethi JK and Hotamisligil GS: The role of TNF alpha in adipocyte metabolism. Semin Cell Dev Biol 10: 19-29, 1999.

24. Wernstedt Asterholm I, Tao C, Morley TS, Wang QA, Delgado-Lopez F, Wang ZV and Scherer PE: Adipocyte inflammation is essential for healthy adipose tissue expansion and remodeling. Cell Metab 20: 103-118, 2014.

25. Lumeng CN and Saltiel AR: Inflammatory links between obesity and metabolic disease. J Clin Invest 121: 2111-2117, 2011. 